\title{
Dabrafenib desensitization after a vemurafenib-induced hypersensitivity reaction in a pediatric patient with BRAFV600E mutant pilocytic astrocytoma
}

\author{
Melissa Bourque ${ }^{1}$, Meredith Canale ${ }^{1}$, Noah Sabin ${ }^{2}$, Marta Salek ${ }^{1}$, and Santhosh \\ Upadhyaya $^{1}$ \\ ${ }^{1}$ Saint Jude Children's Research Hospital \\ ${ }^{2}$ St. Jude Children's Research Hospital
}

October 16, 2020

\begin{abstract}
BRAFV600E inhibitor therapy, either alone or in combination with a MEK inhibitor, demonstrates positive responses in pediatric patients with gliomas harboring the BRAFV600E mutation. However, rare but life-threatening hypersensitivity reactions to BRAFV600E inhibitors may result in abrupt and permanent discontinuation of therapy. We report successful desensitization, tolerance, and continued response to an alternative BRAFV600E inhibitor plus the addition of a MEK inhibitor in a 7-year-old girl with bilateral optic pathway glioma who experienced a severe hypersensitivity reaction to vemurafenib. This strategy thereby avoided the systemic late effects of chemotherapy and radiation therapy.
\end{abstract}

\section{1 - INTRODUCTION}

$B R A F^{V 600 E}$ mutations occur in $5 \%$ to $16 \%$ of pediatric patients with pilocytic astrocytoma, which is the most common type of pediatric brain tumor. This alteration is associated with high recurrence rates, despite conventional chemotherapy, and poor progression-free survival. ${ }^{1-3}$ The $\mathrm{BRAF}^{\mathrm{V} 600 \mathrm{E}}$ inhibitors vemurafenib and dabrafenib have demonstrated considerable promise for pediatric patients with relapsed/refractory gliomas harboring the $B R A F^{V 600 E}$ mutation. ${ }^{4}$ However, hypersensitivity reactions, such as cutaneous reactions to $\mathrm{BRAF}^{\mathrm{V} 600 \mathrm{E}}$ inhibitors, are common, even in pediatric patients. ${ }^{5,6}$ If cutaneous reactions are severe, this leads to permanent discontinuation of a potentially life-saving therapy. Vemurafenib and dabrafenib share similar chemical structures, including a sulfonamide group. ${ }^{5}$ Cross-reactivity between BRAF inhibitors and other sulfonamides may contribute to their intolerance. ${ }^{5}$ Hypersensitivity reactions to $\mathrm{BRAF}^{\mathrm{V} 600 \mathrm{E}}$ inhibitors followed by tolerance to another $\mathrm{BRAF}^{\mathrm{V} 600 \mathrm{E}}$ inhibitor is rarely described, especially for pediatric patients. To date, successful transition from one BRAFV ${ }^{600 \mathrm{E}}$ inhibitor to another is reported in only one pediatric case of anaplastic ganglioglioma and in three adult cases of melanoma. ${ }^{7-10}$ Here, we describe an immediate hypersensitivity reaction to vemurafenib in a pediatric patient with a pilocytic astrocytoma. We then administered a desensitization regimen with dabrafenib and trametinib, which yielded a positive tumor response without further allergic reaction.

\section{2 - CASE PRESENTATION}

We describe a now 9-year-old female patient with an optic pathway glioma diagnosed by magnetic resonance imaging at 5 months old after experiencing bilateral nystagmus (Figure 1A and B). A genetic work up ruled out the presence of neurofibromatosis-1. Because of the definitive imaging features consistent with optic pathway glioma, she was administered vincristine and carboplatin at the referring institution without biopsy. ${ }^{11}$ After 9 months of treatment, she experienced a carboplatin allergy and was subsequently switched 
to weekly vinblastine to complete 1 year of therapy. After 18 months, imaging findings revealed progressive disease (PD), and she was administered bevacizumab and irinotecan, with frequent treatment interruptions due to proteinuria. ${ }^{12}$ At 5 years old, she received a tumor biopsy, which confirmed pilocytic astrocytoma with a $B R A F^{V 600 E}$ mutation, and was subsequently enrolled in a clinical trial using trametinib. She was removed from the trial after 7 months because of PD. She was followed with serial imaging and eye exams for 1 year and then referred to St. Jude Children's Research Hospital for consideration of targeted therapy for her tumor when visual worsening was noted on an ophthalmological exam (Figure 1C and D).

Upon presentation to St. Jude Children's Research Hospital at 7 years old, she exhibited bilateral nystagmus and visual field constriction on confrontation testing but no motor or other cranial nerve deficits. Ophthalmological evaluation revealed a visual acuity of 20/200 and 20/100 bilaterally for distance and near vision, respectively (Supporting Information Table S1). A visual field examination was remarkable for bilateral superotemporal quadrantanopia. She was administered a targeted therapy regimen with single-agent vemurafenib but experienced a maculopapular rash over her face, trunk, and extremities after 2 weeks, warranting discontinuation. The rash resolved over the next 2 weeks, and vemurafenib was re-administered at a reduced dose. However, within 3 hours of administering vemurafenib, she experienced an extensive full body rash with intense itching. Because of the likelihood of an anaphylactic reaction occurring with further vemurafenib administration and after discussion with her family, vemurafenib was permanently discontinued.

Reports primarily from adult studies suggest that using either dabrafenib alone or in combination with a MEK inhibitor, such as trametinib, can reduce the risk of skin toxicity. ${ }^{8-10}$ This treatment strategy was presented to the family, and they decided to proceed with dabrafenib and trametinib treatment. Because of her prior hypersensitivity reaction to vemurafenib, we formulated a desensitization plan with slow escalation and administered dabrafenib at $35 \%$ of the recommended dose concurrently with full-dose trametinib (Table 1). She was observed in the hospital daily for 6 hours after dabrafenib administration during the first week of therapy. No adverse events were observed, and the dabrafenib dose was slowly escalated over the next 3 weeks.

Apart from rare interruptions of therapy for fever symptoms, both medications were tolerated without any dose reductions for approximately 18 months. Ophthalmological examinations revealed improved near vision (20/60 OS and 20/70 OD) with stable distant vision (Supporting Information Table S1). The tumor demonstrated an immediate response, with decreased enhancement and size of the lesion within 8 weeks of starting treatment (Figure $1 \mathrm{E}$ and F), and continues to show slow, but steady improvement after 18 months of therapy (Figure $1 \mathrm{G}$ and $\mathrm{H}$ ).

\section{3 - DISCUSSION}

BRAF inhibitors were originally approved to treat melanomas with the $B R A F^{V 600 E}$ mutation after demonstrating improved survival in patients with unresectable or metastatic melanoma. ${ }^{5,13}$ In pediatric gliomas harboring a $B R A F^{V 600 E}$ alteration, BRAF ${ }^{\mathrm{V} 600 \mathrm{E}}$ inhibitors not only produce dramatic imaging responses but also may halt or reverse tumor-induced functional impairments. ${ }^{4,14}$ Despite the promising efficacy of BRAF inhibitors in a variety of $B R A F$-mutant cancers, dabrafenib or vemurafenib frequently cause intolerable adverse effects, such as severe cutaneous reactions. ${ }^{13}$

A patient with vemurafenib-induced toxic epidermal necrolysis (TEN) experienced successful transition from vemurafenib to dabrafenib by slowly increasing dabrafenib over 1 week to the maximum adult dose without a return of toxicity. ${ }^{8}$ Another patient experienced a hypersensitivity reaction to vemurafenib and after 2 weeks was administered dabrafenib. ${ }^{9}$ However, the patient experienced a hypersensitivity reaction, including generalized urticaria with angioedema, within 1 hour after the second dose of dabrafenib. ${ }^{9}$ Because of PD, the patient received a desensitization protocol with escalating doses of dabrafenib, in addition to premedication prednisone and promethazine. The patient tolerated the desensitization and was able to receive full-dose dabrafenib without further adverse effects. ${ }^{9}$

Cross-reactivity between vemurafenib and dabrafenib and other sulfonamides may contribute to intolerance. ${ }^{5}$ A patient who experienced vemurafenib-induced TEN did not continue further dabrafenib treatment be- 
cause a lymphocyte transformation test confirmed cross-reactivity to sulfamethoxazole, vemurafenib, and dabrafenib. ${ }^{5}$ However, in vitro and skin test studies suggest that cross-reactivity with sulfonamide-containing antibiotics and non-antibiotics is unlikely. ${ }^{15}$

Combination therapy with a MEK inhibitor, such as trametinib or cobimetinib, increase progression-free survival and overall survival by decreasing resistance and cutaneous reactions in patients with melanoma. ${ }^{7,16-18}$ Only one pediatric study has reported tolerance and response to a dabrafenib and trametinib combination therapy in a patient with previous vemurafenib intolerance. ${ }^{7}$ The patient experienced pyrexia and rigors upon treatment with dabrafenib, but these symptoms resolved by withholding therapy for 24 hours, and the patient experienced disease resolution without toxicity. ${ }^{7}$ In addition, an adult patient who experienced vemurafenib-induced TEN tolerated a gradual dose escalation of dabrafenib in combination with trametinib and corticosteroids. ${ }^{10}$ Over 17 weeks, the dose and frequency of dabrafenib were increased with daily trametinib and prednisone. ${ }^{10}$ This desensitization approach allowed successful dabrafenib transition without further cutaneous reactions. ${ }^{10}$

The patient we describe here experienced a cutaneous reaction during her first course of vemurafenib and within 3 hours of receiving a reduced dose. Radiation therapy was considered, but because of her young age and the potential for long-term neurocognitive and endocrine adverse effects, a desensitization approach with dabrafenib in addition to trametinib was pursued to prevent recurring cutaneous or more severe allergic reactions. She not only tolerated the dose escalation without antihistamines or corticosteroids but also has had no cutaneous reactions. She continues to experience a positive tumor response and improved vision.

\section{CONFLICTS OF INTEREST}

The authors declare no conflicts of interest.

\section{ETHICAL STATEMENT}

Informed consent was obtained.

\section{ACKNOWLEDGMENTS}

The authors gratefully acknowledge Nisha Badders, PhD, ELS, for editing the manuscript.

\section{REFERENCES}

1. Rickert CH, Paulus W. Epidemiology of central nervous system tumors in childhood and adolescence based on the new WHO classification. Child's Nerv Syst. 2014;17(9):503-511.

2. Maraka S, Janku F. BRAF alterations in primary brain tumors.Discov Med. 2018;26:51-60.

3. Kurani H, Gurav M, Shetty O, et al. Pilocytic astrocytomas: BRAFV600E and BRAF fusion expression patterns in pediatric and adult age groups. Child's Nerv Syst. 2019;35(9):1525-1536.

4. Hargrave DR, Bouffet E, Tabori U, et al. Efficacy and safety of dabrafenib in pediatric patients with BRAF V600 mutation-positive relapsed or refractory low-grade glioma: results from a phase I/IIa study. Clin Cancer Res. 2019;25(24):7303-7311.

5. Bellón T, Lerma V, González-Valle O, González Herrada C, J de Abajo F. Vemurafenib-induced toxic epidermal necrolysis: possible cross-reactivity with other sulfonamide compounds. $\mathrm{Br} J$ Dermatol . 2016;174(3):621-624.

6. Song H, Zhong CS, Kieran MW, Chi SN, Wright KD, Huang JT. Cutaneous reactions to targeted therapies in children with CNS tumors: A cross-sectional study. Pediatr Blood Cancer . 2019;66(6):e27682.

7. Marks AM, Bindra RS, DiLuna ML, et al. Response to the BRAF/MEK inhibitors dabrafenib/trametinib in an adolescent with a BRAF V600E mutated anaplastic ganglioglioma intolerant to vemurafenib.Pediatr Blood Cancer. 2017;65(5):e26969.

8. Jeudy G, Dalac-Rat S, Bonniaud B, et al. Successful switch to dabrafenib after vemurafenib-induced toxic epidermal necrolysis. Br J Dermatol. 2015;172(5):1454-1455.

9. Bar-Sela G, Abu-Amna M, Hadad S, Naim N, Shahar E. Successful desensitization protocol for hypersensitivity reaction probably caused by dabrafenib in a patient with metastatic melanoma. Jpn J Clin 
Oncol . 2015;45(9):881-883.

10. Tahseen AI, Patel NB. Successful dabrafenib transition after vemurafenib-induced toxic epidermal necrolysis in a patient with metastatic melanoma. JAAD Case Reports . 2018;4(9):930-933.

11. Packer RJ, Ater J, Allen J, Phillips P, Geyer R, Nicholson HS, Jakacki R, Kurczynski E, Needle M, Finlay J, Reaman G, Boyett JM. Carboplatin and vincristine chemotherapy for children with newly diagnosed progressive low-grade gliomas. J Neurosurg. 1997;86(5): 747-54.

12. Packer, Jakacki R, Horn M, et al. Objective response of multiply recurrent low-grade gliomas to bevacizumab and irinotecan. Pediatr Blood Cancer . 2009;52(7):791-795.

13. Croce L, Coperchini F, Magri F, Chiovato L, Rotondi M. The multifaceted anti-cancer effects of BRAFinhibitors. Oncotarget . 2019;10(61):6623-6640.

14. Upadhyaya SA, Robinson GW, Harreld JH, et al. Marked functional recovery and imaging response of refractory optic pathway glioma to BRAFV600E inhibitor therapy: a report of two cases. Childs Nerv Syst. 2018;34(4):605-610.

15. Zawodniak A, Lochmatter P, Beeler A, Pichler WJ. Cross-reactivity in drug hypersensitivity reacts to sulfasalazine and sulfamethoxazole.Int Arch Allergy Immunol . 2010;153(2):152-156.

16. Larkin J, Ascierto PA, Dréno B, et al. Combined vemurafenib and cobimetinib in BRAF-mutated melanoma. N Engl J Med.2014(20);371:1867-1876.

17. Long GV, Stroyakovskiy D, Gogas H, et al. Combined BRAF and MEK inhibition versus BRAF inhibition alone in melanoma. $N$ Engl $J$ Med. 2014;371(20):1877-1888.

18. Robert C, Karaszewska B, Schachter J, et al. Improved overall survival in melanoma with combined dabrafenib and trametinib. $N$ Engl J Med . 2014;(1)372:30-39.

\section{FIGURE LEGEND}

FIGURE 1 Fat-saturated axial T2 weighted image (A) showing tortuosity and thickening of the intraorbital portions of the optic nerves (arrows) and a postcontrast axial T1-weighted image (B) demonstrating enhancement in and along the enlarged prechiasmatic optic nerves, optic chiasm, and proximal optic tracts (arrows), consistent with an optic pathway glioma at 5 months of age. Postcontrast T2 FLAIR image (C) before receiving targeted therapy at 7 years of age showing expansile hyperintensity in the bilateral optic tracts (arrows) and an increase in abnormal signal in the region of the hypothalamus and anterior commissure (large arrow) with a postcontrast axial T1-weighted image (D) showing increased enhancement in the bilateral optic tracts and in the optic chiasm (arrows). Postcontrast axial T2 FLAIR image (E) showing decreased expansile hyperintensity in portions of the bilateral optic tracts (arrows) and postcontrast axial T1-weighted image (F) demonstrating near complete to complete resolution of enhancement in the optic tracts and optic chiasm (arrows) after 8 weeks of targeted therapy. Postcontrast axial T2 FLAIR image (G) demonstrating continued decreased expansile hyperintensity in the bilateral optic tracts and in the region of the anterior commissure and hypothalamus (arrows) and a postcontrast axial T1-weighted image $(\mathrm{H})$ showing resolution of enhancement in the optic tracts (arrows) after 18 months of targeted therapy.

\section{Hosted file}

Dabrafenib Desensitization TABLE 1--10.14.2020.pdf available at https://authorea.com/ users/367688/articles/487030-dabrafenib-desensitization-after-a-vemurafenib-inducedhypersensitivity-reaction-in-a-pediatric-patient-with-brafv600e-mutant-pilocyticastrocytoma 

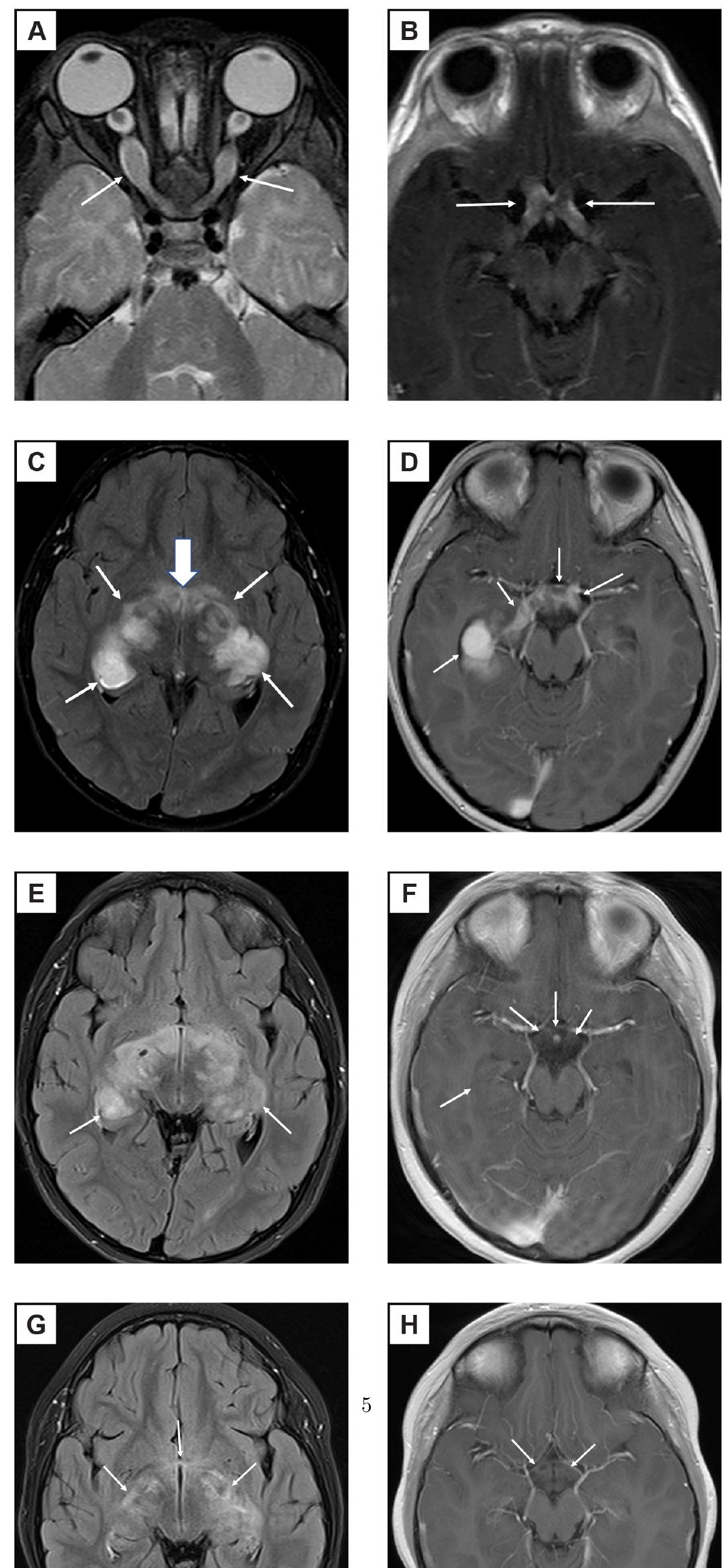